\title{
Berufseinstiege - Promotionen - Kompetenzen: Hochschulen und Absolventen vor neuen Herausforderungen
}

\begin{abstract}
Vor dem Hintergrund des Wandels der Arbeitsgesellschaft gewinnt das Verhältnis zwischen Hochschule und Arbeitsmarkt an Bedeutung. Im Zuge des Bologna-Prozesses wird als Ziel anvisiert, die Beschäftigungsfähigkeit der Hochschulabsolventinnen und -absolventen zu verbessern, indem transferierbare und überfachliche Schlüsselkompetenzen vermittelt werden. Auch im Hinblick auf den wissenschaftlichen Nachwuchs sollten solche Schlüsselkompetenzen an Bedeutung gewinnen. Der Beitrag untersucht anhand des Absolventenpanels des Hochschul-Informations-Systems (HIS-Absolventenpanel) zum einen, wie sich die Übergänge in angemessene Beschäftigung und Promotionen entwickelt haben. Zum anderen wird der Stand bzw. die Veränderung von Kompetenzprofilen und Kompetenzanforderungen analysiert.
\end{abstract}

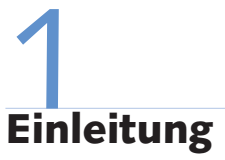

Die Phase des Berufseinstiegs gilt als einer der zentralen Indikatoren für den frühen Berufserfolg und als ein Evaluationskriterium für die Qualität der hochschulischen Ausbildung (Wissenschaftsrat 1999). Im Vergleich zu Absolventinnen und Absolventen einer dualen Berufsausbildung, deren Berufseinstiege aufgrund ihrer betrieblichen Anbindung und relativ hoher Übernahmequoten einem zeitlich eher reibungslosen Ablauf folgen (Falk et al. 2000), gestalten sich Berufseinstiege von Hochschulabsolventinnen und -absolventen heterogener.

Für viele Akademiker ist der Berufseinstieg eher als sequenzieller Prozess mittlerer Reichweite zu verstehen. Ein Teil der Absolventen hat nach dem Studienabschluss mindestens eine weitere Schwelle zu bewältigen. Vorbereitungsdienste oder Promotionsaktivitäten beschreiben Phasen typischer Berufseinstiegssequenzen beziehungsweise typische Einmündungswege in den - hinsichtlich des Prestigeoder Einkommensniveaus - vollwertigen Beruf. Während in Fächern mit integralen Ausbildungsbestandteilen der Berufseinstiegsprozess mit den Anforderungen eines zweiten Staatsexamens weitgehend vorgezeichnet ist, stehen in anderen Fächergruppen individuelle Entscheidungsprozesse zum Beispiel für oder gegen eine Tätigkeit im Wissenschaftssystem an. Diese werden in aller Regel auch von der Entscheidung für oder gegen eine Promotion begleitet.
Einigkeit besteht hinsichtlich der Annahme, dass der Übergang vom Studium in den Beruf vor dem Hintergrund des Wandels der Arbeitsgesellschaft und veränderter Qualifikationsanforderungen (Abschnitt 2) „schwieriger, risikoreicher und langwieriger" (Wissenschaftsrat 1999, S. 6) geworden ist. Dabei wird davon ausgegangen, dass die Ausbildung des eigenen wissenschaftlichen Nachwuchses und die erfolgreiche Vermittlung der nicht im akademischen Bereich verbleibenden Absolventen nur über die Sicherung der Studienqualität bzw. die Verbesserung der Beschäftigungsfähigkeit mittels einer passgenaueren Vermittlung von Kompetenzen gelingen kann. Wenn es richtig ist, dass zukünftige Wachstumschancen in hohem Maße auf einer innovations- und wissensbasierten Ökonomie beruhen (Krull 2008), ist der quantitativen Entwicklung von Promotionen bei der Untersuchung von Berufseinstiegen besondere Aufmerksamkeit zu schenken (Abschnitt 3).

Hinsichtlich des Zugangs zu wissenschaftlichen Karrieren ist aber von Chancenungleichheiten auszugehen (Leemann 2002), die neben offensichtlichen fächerkulturellen Unterschieden auf individuelle Fähigkeiten und Merkmale wie das Geschlecht oder die Bildungsherkunft sowie Merkmale des Studienverlaufs zurückzuführen sein sollten. Fraglich ist dabei, inwieweit neben demografischen Entwicklungen auch die Reformierung von Studiengängen im Rahmen des BolognaProzesses den Übergang in die Beschäftigung/Promotion beeinflusst und Kompetenzprofile im Hinblick auf die späteren Kompetenzanforderungen begünstigt.
Diesem Problem widmet sich Abschnitt 4. Abschnitt 5 fasst die Befunde zusammen.

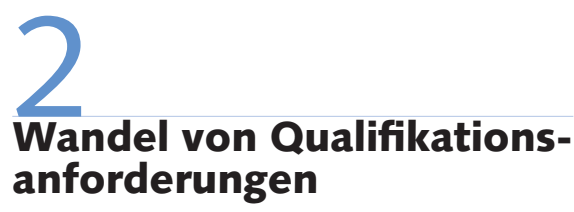

Vor dem Hintergrund zunehmender Disparitäten sowie struktureller Veränderungen von Arbeitsangebot und -nachfrage werden seit Langem weitreichende Veränderungen von Qualifikationsanforderungen am Arbeitsmarkt konstatiert. Exemplarisch werden jene Entwicklungsprozesse unter den Stichworten der Tertiarisierung und Informatisierung, der Erosion des Normalarbeitsverhältnisses oder des Wandels der Arbeits- und Betriebsorganisation behandelt. Die Globalisierung kann als ein Katalysator verstanden werden, der die genannten Prozesse und einen Wandel von Qualifikationsanforderungen beschleunigt.

Beispielsweise entsteht im Rahmen der Tertiarisierung eine Vielzahl höherwertiger Dienstleistungstätigkeiten, die große Autonomie bezüglich der Arbeits- und Kooperationsgestaltung, der Zeitverwendung und der räumlichen Mobilität ermöglichen oder verlangen (Baethge/Baethge-Kinsky

Michael Grotheer, M.A. Soziologie,
wissenschaftlicher Mitarbeiter an der
Hochschul-Informations-GmbH (HIS),
Hannover. Arbeitsschwerpunkte:
Sozialstrukturanalyse, Arbeitsmarkt- und
Lebenslaufforschung, Absolventenforschung.
e-mail: grotheer@his.de


1998). Neue Formen der Arbeitsorganisation werden auch im Bereich innovationsund wissensintensiver Unternehmen z. B. über Zielvereinbarungen, Gruppen-/ Projektarbeit, Job-Enrichment oder betriebliche Weiterbildung dokumentiert (Picot et al. 2001). Diesen qualitativen Formen der Flexibilität stehen quantitative Maßnahmen gegenüber (Goudswaard/ Nanteuil 2000), die Anpassungen des Arbeitsvolumens an eine wechselnde Nachfrage beschreiben und Ausdruck in einer zunehmenden Zahl atypischer Beschäftigungsverhältnisse finden (Befristung, Selbstständigkeit, flexible Arbeitszeitmodelle), die auch für Hochschulabsolventinnen und Hochschulabsolventen durchaus nicht unüblich sind (Briedis 2007).

Immer größere Bedeutung wird in diesen Kontexten jenen von den herkömmlichen Fachkompetenzen substanziell zu unterscheidenden Schlüsselqualifikationen wie Sozial- oder (Selbst-)Organisationskompetenzen beigemessen, die sich z.B. auf die Fähigkeit beziehen, sich an kooperativen Arbeitsformen zu beteiligen, das berufliche Leben aktiv zu gestalten, oder auf die Begabung, sich auf veränderte Umstände einstellen und Verantwortung übernehmen zu können (Schaeper/Briedis 2004; Orth 1999). Solche Schlüsselqualifikationen sollten vor allem für jene Personen einen höheren Stellenwert haben, die in team- oder kundenorientierten sowie innovativen und eigenverantwortlichen Arbeitszusammenhängen wirtschaftlich ausgerichteter Unternehmen tätig sind. Wenngleich Promovierende sich häufiger in projektorientierten kundenfernen Arbeitszusammenhängen befinden, lassen einige Entwicklungen auch für den wissenschaftlichen Arbeitsmarkt eine wachsende Bedeutung der Schlüsselkompetenzen vermuten. Diese lassen sich unter den Stichworten einer Professionalisierung der Wissenschaft, eines zunehmenden Wettbewerbs um Drittmittel, der in einigen Fächergruppen verbreiteten Forschung in Teams und größeren Projektzusammenhängen, der Internationalisierung von Ausbildungsstrukturen oder der Ausweitung strukturierter Formen der Doktorandenausbildung zusammenfassen (Berning/Falk 2005).

\section{Berufseinstiege von Hoch- schulabsolventen}

Der Stellenwert der beruflichen Erstplatzierung für berufliche Verläufe wurde in der soziologischen Arbeitsmarkt- und Lebenslaufforschung seit den späten 1970er Jahren zunächst anhand von Fragestellungen zum intergenerationalen Wandel thematisiert. Dabei wurde festgestellt, dass die Vor- oder Nachteile, die bestimmte Abschlusskohorten beim Eintritt in das Beschäftigungssystem erfahren, sich auch nach Jahrzehnten im Beschäftigungssystem noch widerspiegeln (Blossfeld 1989). Auch in einer intragenerationalen Perspektive bestätigt eine Vielzahl von Untersuchungen die langfristigen Wirkungen positiver und negativer Erfahrungen auf dem Arbeitsmarkt. Sie können den weiteren Lebenslauf der Personen beeinflussen oder prägen (Grotheer 2008; Falk et al. 2000; OECD 1998). Obgleich der Berufseinstieg vor allem für Promovierende oder Personen aus Studiengängen mit anschließenden Vorbereitungsdiensten eher als ein mittelfristiger Prozess denn als ein zeitpunktbezogener Übergang zu verstehen ist, wird der Begriff des Berufseinstiegs in der Regel unabhängig von der Art der Tätigkeit als Erstplatzierung auf dem Arbeitsmarkt nach Abschluss einer (ersten) beruflichen Bildungsphase definiert (Dietrich/Abraham 2005).

\subsection{DATEN UND METHODEN}

Grundlage für die Analyse der Entwicklung von Berufseinstiegen und Kompetenzen sind die Daten der Erstbefragungen verschiedener Jahrgänge von Hochschulabsolventinnen und -absolventen aus dem HIS-Absolventenpanel. ${ }^{1}$ Dabei hat sich in früheren Analysen zum Thema Berufseinstiege eine Perspektive als ertragreich erwiesen, die beim Übergang von der Hochschule in den Arbeitsmarkt klare Unterschiede im Niveau der Beschäftigung berücksichtigt. Dementsprechend werden Übergänge in Beschäftigung auch hier nur dann als Berufseinstiege gewertet, wenn es sich um ein der Qualifikation angemessenes Beschäftigungsverhältnis handelt (Grotheer 2009; 2010). ${ }^{2}$

Im Gegensatz zur Erwerbstätigkeit ist die genaue Bestimmung des Zeitpunktes der Aufnahme einer Promotion schwieriger. Zum einen besteht keine generelle Pflicht zur Einschreibung für Doktoranden. Auch sind der eigentlichen Arbeit an der Promotion Phasen wie die Themensuche und Themenvorbereitung oder die Suche nach Betreuern und einer geeigneten institutionellen Struktur vorangestellt. Dennoch gibt der Großteil der Personen an, die Promotion innerhalb eines Jahres nach Studienabschluss aufgenommen zu haben (Kerst/Schramm 2008, S. 44). Auch wenn diese Gruppe aufgrund von Abbrechern oder Nachzüglern nicht ganz deckungsgleich mit den tatsächlich realisierten Promotionen ist (ebd., S. 158), spiegelt sie dennoch in etwa das Promotionspotenzial einer Fächergruppe. In den Auswertungen wird dabei zwischen Promotionen unterschieden, die entweder parallel zu angemessener Beschäftigung stattfinden (in der Regel Promotionsstellen) oder aber über andere Quellen finanziert werden (Stipendien, Graduiertenförderung, Jobben, Kredite oder sonstige Einkünfte). Für die Darstellungen wird eine Auswahl von Fächergruppen/Studienbereichen an Universitäten getroffen, die sowohl hinsichtlich der Stichprobengröße als auch der Übergänge in Promotionen ausreichende Fallzahlen aufweisen.

1 Beim HIS-Absolventenpanel handelt es sich um eine schriftliche Befragung von Erstabsolventinnen und -absolventen eines Hochschulstudiums, in der retrospektiv bundesweit repräsentative Daten nach Fachrichtungen, Hochschul- und Abschlussarten sowie für regionale Einheiten erhoben werden (Kerst/Schramm 2008; Briedis 2007). Im Rahmen des Paneldesigns wird seit 1989 alle vier Jahre ein neuer Abschlussjahrgang aufgenommen, dessen Erstbefragung etwa ein Jahr nach Studienabschluss stattfindet. Dabei werden u. a. ausführliche Informationen zum Studienverlauf, zum Berufseinstieg und zur beruflichen Situation sowie zu Phasen der wissenschaftlichen Weiterqualifizierung erhoben. Um auch die längerfristigen Entwicklungen erfassen zu können, findet eine zweite Befragung der Personen etwa fünf Jahre nach Abschluss statt (Kerst/Schramm 2008). Für den Jahrgang von 1997 konnte außerdem eine dritte Befragung zehn Jahre nach Abschluss realisiert werden (Fabian/Briedis 2009). Sämtliche Fragebögen stehen ab dem Jahrgang 1997 auf der HIS-Homepage zur Einsicht bereit (http://www.his.de/abt2/ab22/fragebogen/)

2 Die Angemessenheit einer Beschäftigung wird aus der beruflichen Stellung abgeleitet. Abhängige Erwerbstätigkeiten als "un- und angelernte Arbeiter, Facharbeiter, ausführende Angestellte oder mithelfende Familienangehörige" wurden ebenso als unterwertig bezeichnet wie "abhängig oder selbstständig Beschäftigte mit einer Wochenarbeitszeit von weniger als zehn Stunden". 


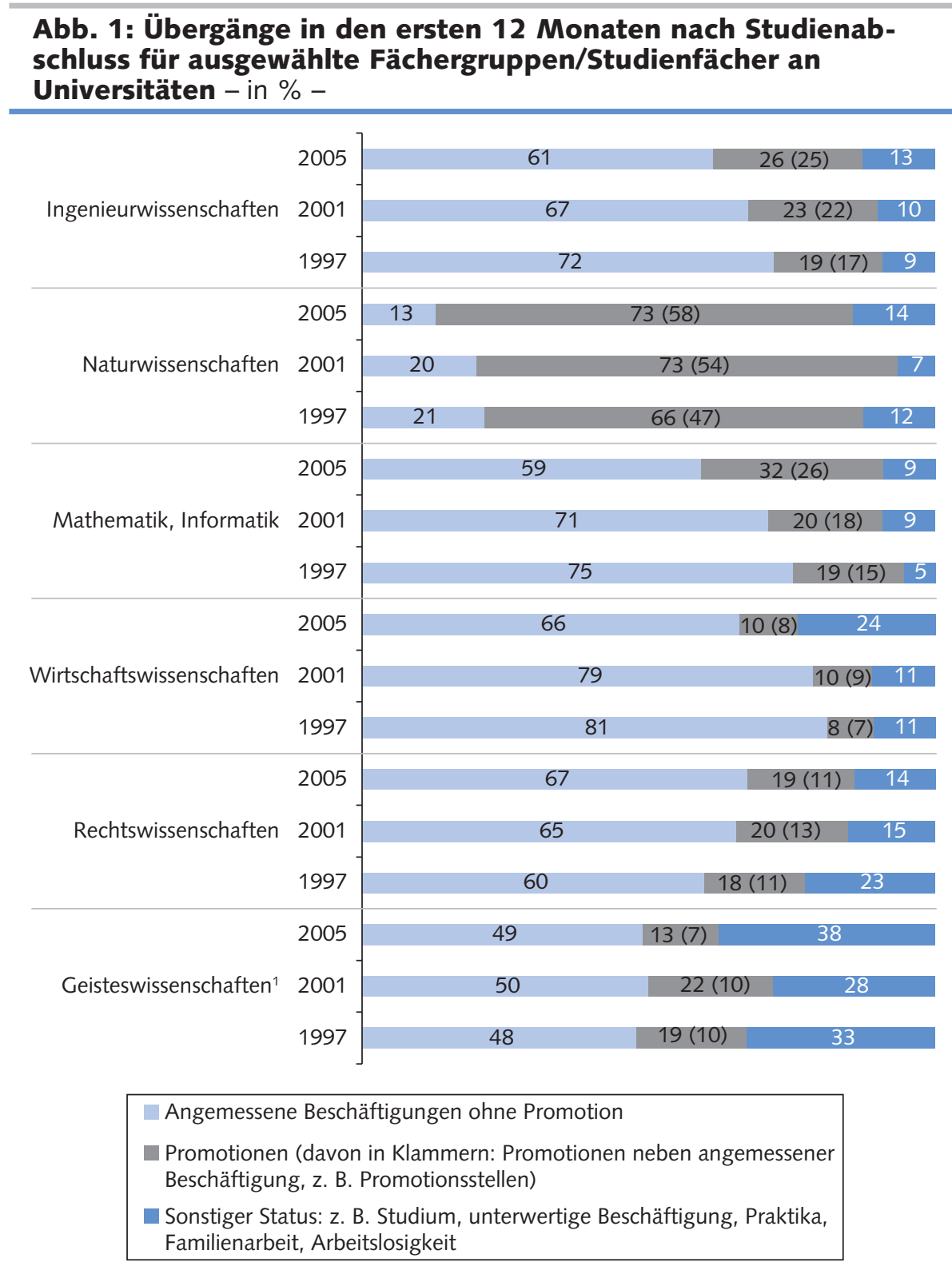

1) Geisteswissenschaften umfassen im Sinne der Definition des Wissenschaftsrates (2006, S. 17) und gemäß der Codierung des Statistischen Bundesamtes (VIB-Hochschulstatistik: Stand 2009) alle Studienfächer der Fächergruppe Sprach- und Kulturwissenschaften sowie der Fächergruppe Kunst und Kunstwissenschaften.

\section{2 ÜBERGÄNGE IN ANGEMESSENE BESCHÄFTIGUNG UND PROMOTION}

Abbildung 1 verdeutlicht die Einstiegschancen von Universitätsabsolventinnen und -absolventen traditioneller Studiengänge verschiedener Abschlussjahrgänge. Wenngleich erhebliche Fächergruppenunterschiede und unterschiedliche Risikoverteilungen im Zeitverlauf bestehen, kann man insgesamt davon sprechen, dass der Arbeitsmarkt für hochqualifizierte Arbeitnehmer durchaus in der Lage ist, die Absolventen der verschiedenen Jahrgänge aufzunehmen. Einem Großteil der Personen ist innerhalb der ersten zwölf Monate nach Abschluss der Übergang in eine angemessene Beschäftigung oder die Aufnahme einer Promotion gelungen. Abgesehen vom Bereich der Naturwissenschaften dominiert der Status angemessener Beschäftigung ohne Promotion.

Auch hinsichtlich der Promotionsaktivität und der Promotionsformen sind beträchtliche Fächergruppenunterschiede zu beobachten. Spitzenreiter ist der Bereich Naturwissenschaften, in dem über 70\% der Absolventen eine Promotion begon- nen haben. Davon finden etwa drei Viertel der Promotionen parallel zu angemessener Beschäftigung und in der Regel auf Promotionsstellen statt. Das restliche Viertel der Promovierenden dieser Fächergruppe bedient sich anderer Finanzierungsquellen (z.B. Stipendien, Jobben, private Einkünfte).

Den zweitgrößten Anteil begonnener Promotionen weisen die Bereiche Ingenieurwissenschaften und Mathematik/Informatik auf. Ingenieurwissenschaftlerinnen und -wissenschaftler promovieren dabei fast ausschließlich parallel zu angemessener Beschäftigung (25 von 26 Doktoranden des Abschlussjahrgangs 2005) und am häufigsten im Rahmen einer Vollzeitstelle (Grotheer 2010). Sowohl Ingenieur- als auch Naturwissenschaftler erarbeiten ihre Promotionen oftmals im Rahmen eines größeren Forschungsvorhabens und in engem Kontakt zu anderen Wissenschaftlern, während in den Bereichen Rechts- oder Geisteswissenschaften die Alleinbearbeitung die am häufigsten genannte Form der Promotion darstellt (Kerst/Schramm 2008, S. 164). Bei Letzteren findet nur etwa die Hälfte der Promotionen parallel zu angemessener Beschäftigung statt.

Im Kohortenvergleich ist die Neigung zur Aufnahme einer Promotion zwischen 1997 und 2001 in allen Fächergruppen gestiegen, im Bereich der Naturwissenschaften um ganze sieben Prozentpunkte. Zwischen 2001 und 2005 sind unterschiedliche Entwicklungen festzustellen. Vor allem im Bereich Mathematik/Informatik steigt der Anteil begonnener Promotionen von etwa $20 \%$ auf nunmehr fast ein Drittel der Absolventinnen und Absolventen an. Auch im Bereich Ingenieurwissenschaften erhöhen sich die Anteile Promovierender geringfügig. Konstante Anteile zeigen sich hingegen in den Bereichen Naturwissenschaften und Wirtschaftswissenschaften. Rückläufig sind die Anteile bei den Rechtswissenschaften und vor allem im Bereich der Geisteswissenschaften, in dem sie sogar deutlich unter das Niveau von 1997 fallen. Weitere Bildungsinvestitionen scheinen somit für Geisteswissenschaftler keine alternative Option zur Überbrückung der zu diesem Zeitpunkt eher ungünstigen Arbeitsmarktlage zu sein.

Neben den hier dargestellten Differenzen zwischen den Fächergruppen und Jahrgängen bestehen weitere (fächerübergreifende) Ungleichheiten. Die Ergebnisse früherer Analysen zum Thema Berufseinstiege (Grotheer 2009, 2010) belegen, dass 
auch im Bereich akademisch qualifizierter Personen erhebliche geschlechtsspezifische Ungleichheiten vorhanden sind. Frauen sind zum einen auch bei nahezu identischer Qualifikation hinsichtlich der Beschäftigungschancen benachteiligt. Diese Ungleichheiten verschärfen sich im Hinblick auf die Besetzung höherwertiger Beschäftigungsverhältnisse. Zum anderen weisen Frauen weitaus geringere Chancen auf Übergänge in Promotionen auf. Wie die Zahlen der offiziellen Hochschulstatistik zu Habilitationen und Professuren belegen, werden sich die starken Benachteiligungen bezüglich der Promotionschancen im weiteren Karriereverlauf von Frauen noch verstärken.

Des Weiteren zeigt sich eine Fortsetzung der sozialen Selektivität beim Zugang zu Bildung. Die Chancenungleichheit bei der Aufnahme eines Studiums zugunsten von Personen aus einem akademischen Elternhaus (Isserstedt et al. 2007, S. 125ff.) wird bei den Übergängen in Promotionen fortgesetzt und die Bildungsungleichheit dadurch weiter verstärkt. Dabei zeigt sich, dass Personen aus einem akademischen Elternhaus im Durchschnitt mit etwas besseren Examensnoten abschneiden, die wiederum positive Wirkungen auf Übergänge in angemessene Beschäftigung, vor allem jedoch - auch im Zusammenhang der Vergabe von Stipendien - auf Übergänge in Promotionen ausüben.

Die häufig im Rahmen der Diskussion um eine Verbesserung der Studienorganisation vorgebrachte Argumentation, die Studiendauer sei „ein entscheidendes Kriterium beim Berufseinstieg" (Wissenschaftsrat 2005, S. 12), ist empirisch hingegen kaum nachvollziehbar, zumal bei der Definition von Berufseinstiegen der Promotionsstatus in aller Regel keine Rolle spielt:

(1) Hinsichtlich der Übergänge in angemessene Beschäftigung ohne Promotion ist kein negativer Einfluss längerer Studiendauern festzustellen. Beispielsweise geht die fachnahe Beschäftigung während des Studiums, die stark positive Effekte auf Übergänge in angemessene Beschäftigung ausübt, aufgrund des Koordinationsaufwandes in aller Regel mit einer verlängerten Studiendauer einher. Doch auch jenseits der fachnahen Beschäftigung bestehen entgegen der öffentlichen Diskussion keine zwingenden Belege, dass Absolventen mit längeren Studiendauern weniger erfolgreich in den Arbeitsmarkt einsteigen.
(2) Hingegen weisen Absolventen mit kürzeren fachspezifischen Studiendauern durchaus eine höhere Neigung zur Promotion auf. Das erklärt sich partiell durch die etwas besseren Examensnoten dieser Personengruppe und ein geringeres Alter. Ältere Absolventen konzentrieren sich demnach eher auf die Nutzung der verbleibenden Beschäftigungszeiten als auf weitere Bildungsinvestitionen. Die Ergebnisse weisen auf die Notwendigkeit differenzierterer Hypothesen zur Wirkung von Studiendauern hin.

Die These eines zunehmend risikoreichen Berufseinstiegs lässt sich trotz der noch immer privilegierten Stellung von Hochschulabsolventen insgesamt bestätigen. Neben einer zunehmenden Dauer bis zum Eintritt in angemessene Beschäftigung zeigen sich bei der Berücksichtigung von Einkommens-, Aufstiegs- und Zufriedenheitsaspekten weitere Risikodimensionen für einzelne Fächergruppen (Grotheer 2010). Als positiv hingegen sind die im Zeitverlauf zunehmenden Anteile von Personen zu bewerten, die eine Promotion aufgenommen haben, da Vorteile hinsichtlich der zuvor genannten Dimensionen des Berufserfolgs zu erwarten sind. Diese sollten sich aufgrund der länger andauernden Berufseinstiegsprozesse von Doktoranden aber vorrangig in einem späteren Stadium der Karriere (post-doc-Phase) offenbaren.

Trotz der insgesamt positiven Entwicklung der Promotionsanteile zwischen 1997 und 2005 bleibt festzuhalten, dass die absolute Zahl der begonnenen Promotionen rückläufig ist, da sich die Absolventenzahlen dieser Fächergruppen in diesem Zeitraum insgesamt um etwa $10 \%$ verringert haben (zur genauen Entwicklung der Absolventenzahlen: Briedis 2007, S. 2). Lediglich im Bereich Mathematik/Informatik hat sich aufgrund einer Zunahme der Absolventen im Jahr 2005 auch die absolute Zahl der begonnenen Promotionen erhöht.

Die weitere Entwicklung der Zahl der Doktoranden wird maßgeblich von der demografischen Entwicklung, der Bildungsbeteiligung und der Durchlässigkeit reformierter Studiengänge bestimmt werden. Zum einen wird sich der erneute Geburtenrückgang zu Beginn der 1990er Jahre bei gleicher Bildungsbeteiligung negativ auf die Zahl der Studienberechtigten auswirken. In den Jahren von 2007 bis 2013 wird dieser Rückgang noch von den Doppeljahrgängen aufgrund der Schulzeitverkürzungen aufgefangen werden. Zum anderen werden mit den zunehmenden Anteilen von Bachelorabsolventen berufsqualifizierende Abschlüsse generiert, die den Übergang in den Arbeitsmarkt ermöglichen, jedoch nicht zur Promotion berechtigen. Auch Zulassungsbeschränkungen für Masterstudiengänge lassen in einigen Fächergruppen wieder auf einen Rückgang der Promotionsaktivitäten schließen. Hinzu kommen auch im Bereich der industrienahen technischen und naturwissenschaftlichen Fächer hohe Abbrecherquoten in den neuen Bachelorstudiengängen (Heublein et al. 2009).

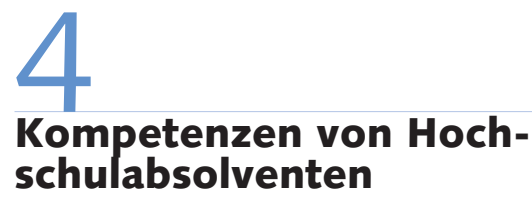

Im Folgenden soll der Blick auf das Verhältnis von Hochschule und Arbeitsmarkt bzw. die Relation zwischen hochschulisch vermittelten und beruflich erforderlichen Kompetenzen gerichtet werden. Nach einer knappen Skizzierung der zu analysierenden Kompetenzdimensionen (Abschnitt 4.1) wird in Abschnitt 4.2 geprüft, ob und wie sich die Kompetenzprofile im Zeitverlauf verändert haben und welchen Einfluss die Reformierung der Studiengänge diesbezüglich ausübt. Den Kompetenzprofilen werden die entsprechenden Einschätzungen zu Kompetenzanforderungen im Berufsleben gegenübergestellt. Fraglich ist dabei, inwieweit vor dem Hintergrund der konstatierten Veränderungen von Qualifikationsanforderungen Differenzen zwischen wissenschaftlich und wirtschaftlich orientierten Arbeitsplätzen Bestand haben.

\subsection{KOMPETENZDIMENSIONEN UND -MESSUNG}

Der Stand der Kenntnisse und Fähigkeiten bei Studienabschluss und die für die berufliche Tätigkeit erforderlichen Kompetenzen werden im HIS-Absolventenpanel mithilfe eines Selbsteinschätzungsinstrumentes erhoben. Die Bewertung von ca. 30 Aspekten erfolgt dabei auf einer Skala von 1 bis 5 (in hohem/geringem Maße vorhanden; sehr wichtig/sehr unwichtig für die Tätigkeit). Zur Reduktion von Komplexität werden analog zu den Arbeiten von Schaeper/Briedis (2004) Dimensionen von Schlüsselkompetenzen genutzt. Einzelne Items wurden dabei mithilfe von Fakto- 


\section{Abb. 2: Stand und Entwicklung der bei Abschluss vorhandenen Kompetenzen von Universitätsabsolventen und -absolventinnen ${ }^{1}$}

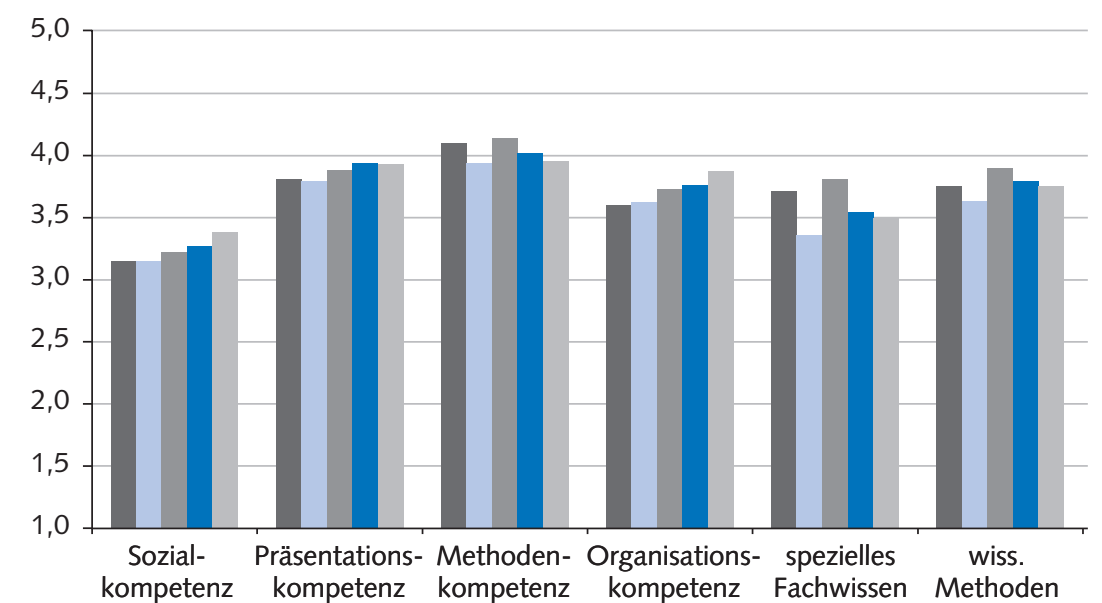

$$
\begin{aligned}
& \text { - Promovierende in angemessener Beschäftigung } 2001 \\
& \text { Angemessen Beschäftigte ohne Promotion } 2001 \\
& \text { Promovierende in angemessener Beschäftigung } 2005 \\
& \text { Angemessen Beschäftigte ohne Promotion } 2005 \\
& \text { Bachelorabsoventen } 2005
\end{aligned}
$$

1) gespiegelte Skala von 1 bis 5 (in geringem bis hohem Maße vorhanden).

ren- und Clusteranalysen zu Kompetenzdimensionen zusammengefasst:

(1) Sozialkompetenzen: Kommunikationsfähigkeit; Verhandlungsgeschick; Führungsqualitäten; Kooperationsfähigkeit; Fähigkeit, Verantwortung zu übernehmen; Konfliktmanagement.

(2) Präsentationskompetenzen: Schriftliche Ausdrucksfähigkeit; Mündliche Ausdrucksfähigkeit.

(3) Methodenkompetenzen: Fähigkeit, Wissenslücken zu erkennen und zu schließen; Fähigkeit, vorhandenes Wissen auf neue Probleme anzuwenden; selbstständiges Arbeiten; Problemlösungsfähigkeit; analytische Fähigkeiten.

(4) (Selbst-)Organisationskompetenzen: Organisationsfähigkeit; Fähigkeit, sich auf veränderte Umstände einzustellen; Zeitmanagement.

(5) Bereichsspezifische Fachkompetenz: Spezielles Fachwissen.

(6) Bereichsspezifische Fachkompetenz: Kenntnis wissenschaftlicher Methoden.
Die aufgelisteten Dimensionen von Schlüsselkompetenzen (1) bis (4) repräsentieren die Durchschnittswerte der jeweils zugeordneten Items. Diese Dimensionen sind zum einen inhaltlich auch in anderen Konzeptualisierungen zu finden (z.B. Orth 1999; Jäger 2001). Zum anderen liegen bereits umfangreiche Analysen zur Güte des Erhebungsinstrumentes und zur Reliabilität der verwendeten Kompetenzdimensionen vor (Schaeper/Briedis (2004), S. 8ff.). Für Fragen nach dem Verhältnis zwischen fachübergreifenden Schlüsselkompetenzen und bereichsspezifischen Fachkompetenzen werden außerdem die zwei Einzelitems (5) und (6) in die Analyse aufgenommen.

\subsection{KOMPETENZPROFILE UND KOMPETENZANFORDERUNGEN}

Wenngleich Studierende mit unterschiedlichen Voraussetzungen an die Hochschulen kommen und auch außerhalb des Fachstudiums - z. B. im Rahmen fachnaher Beschäftigung - Kompetenzen erwerben, zeigt sich hinsichtlich des Standes der Schlüsselkompetenzen bei Studienabschluss ein recht ähnliches Niveau bei Promovierenden und Nicht-Promovierenden
(Abbildung 2). ${ }^{3}$ Lediglich Methodenkompetenzen sind bei Promovierenden etwas höher ausgeprägt. Ein deutlich geringeres Niveau zeigen beide Gruppen bei den Sozialkompetenzen. Auffällige Unterschiede bestehen hingegen bei den bereichsspezifischen Fachkompetenzen wie speziellem Fachwissen und der Kenntnis wissenschaftlicher Methoden, die bei Promovierenden in höherem Maße vorhanden sind.

Nicht verwunderlich ist, dass die bereichsspezifischen Fachkompetenzen für Promovierende auch in höherem Maße erforderlich sind als für Nicht-Promovierende (Abbildung 2 und 3). Der höhere Stand der Fachkompetenzen von Personen mit Promotionsabsichten zeigt aber auch, dass Studierende traditioneller Studiengänge durch Schwerpunktsetzungen innerhalb des Fachcurriculums durchaus in der Lage sind, zusätzliche Potenziale dieser Art aufzubauen. Eine zusätzliche Aneignung von Schlüsselkompetenzen hingegen, von denen vor allem die Sozial- aber auch die Organisationskompetenzen im Berufsleben eine größere Rolle spielen, scheint für diejenigen schwieriger zu sein, die sich auf den Arbeitsmarkt orientieren. Zum einen sind Wirkungen additiver Kurse auBerhalb des Fachcurriculums umstritten (Weinert 1998). Zum anderen sollten von integrativen Ansätzen zur Förderung von Schlüsselkompetenzen alle Studierenden gleichermaßen profitieren.

Bezüglich der Entwicklung der vorhandenen Kompetenzen zeigt sich im Vergleich der traditionellen Absolventen von 2001 und 2005, dass der Kompetenzstand in allen Bereichen leicht zugenommen hat. Die Untersuchungen von Schaeper/Wolter (2008), die anhand derselben Datenquelle den Einfluss von Merkmalen der Studiengestaltung auf den Kompetenzstand analysieren, haben gezeigt, dass insbesondere die Implementierung didaktischer Modelle und Prinzipien (z. B. aktivierende Lehrformen, Projektstudien) zu einer

\footnotetext{
Die Darstellungen der Kompetenzprofile und -anforderungen grenzen den Kreis der Promovierenden auf jene Personen ein, die sich parallel zur Promotion in angemessener Beschäftigung befinden (Promotionsstellen). Da sich die Frage nach den Anforderungen auf die derzeitige (bzw., wenn nicht berufstätig, voraussichtliche) berufliche Tätigkeit bezieht, sind gegenüber Personen, die ihre Promotion z. B. durch unterwertige Beschäftigung oder Förderungen finanzieren, Abweichungen zu vermuten. Der Absolventenjahrgang von 1997 konnte aufgrund einer abweichenden Skala nicht zum Vergleich herangezogen werden.
} 
Verbesserung der Schlüsselkompetenzen, aber auch der Fachkompetenzen beiträgt (ebd., S. 617f.).

Eine Erweiterung des Zeitvergleichs findet quasi mit der Betrachtung des Kompetenzstandes der Bachelorabsolventen von 2005 statt, welche die potenziellen Promovierenden der Folgejahre verkörpern (Abbildung 2). Im Gegensatz zu den Absolventen traditioneller Studiengänge weisen diese ein höheres Niveau an Sozialkompetenzen und an (Selbst-) Organisationskompetenzen auf (so auch Schaeper/Wolter 2008, S. 616f.). Die zeitintensiveren Methodenkompetenzen und bereichsspezifischen Fachkompetenzen werden hingegen in den kürzer angelegten und in der Zielausrichtung „weniger forschungsorientierten Bachelorstudiengängen" (Gensch/Schindler 2003, S. 10) geringer eingeschätzt.

Betrachtet man Stand und Entwicklung der im Berufsleben erforderlichen Kompetenzen (Abbildung 3), so zeigt sich, dass das Niveau der erforderlichen Schlüsselkompetenzen mit einem durchschnittlichen Wert von über vier insgesamt als sehr hoch wahrgenommen wird. Der zum Teil erheblich geringere Stand der Kompetenzen bei Abschluss des Studiums (vor allem bei den Sozialkompetenzen) weist auf die Defizite der Hochschulausbildung hin.

Unterschiede zwischen den Personen mit und ohne Promotionsabsichten bestehen zum einen erwartungsgemäß bezüglich der erforderlichen bereichsspezifischen Fachkompetenzen, deren Stellenwert von den Promovierenden ähnlich hoch wie der der Schlüsselkompetenzen eingeschätzt wird. Zum anderen sind vor allem im Bereich der Sozialkompetenzen Abweichungen erkennbar. Insbesondere in den MINT-Fächern (Mathematik, Informatik, Naturwissenschaften, Technik) sind die Anforderungen an die Sozialkompetenzen Promovierender geringer.

Die Frage nach der Entwicklung der erforderlichen Schlüsselkompetenzen ist aufgrund des kurzen Beobachtungszeitraumes schwieriger zu beantworten. $\mathrm{Zu}$ vermuten ist, dass bereits in den 1980er und 1990er Jahren Steigerungen stattgefunden und zu dem aktuell hohen Niveau geführt haben. Zwischen 2001 und 2005 sind hingegen keine Veränderungen der Anforderungen an die Schlüsselkompetenzen zu beobachten.

Insgesamt zeigt sich, dass die Kompetenzanforderungen beider Personen-

\section{Abb. 3: Stand und Entwicklung der im Berufsleben erforderlichen Kompetenzen von Universitätsabsolventen und -absolventinnen ${ }^{1}$}

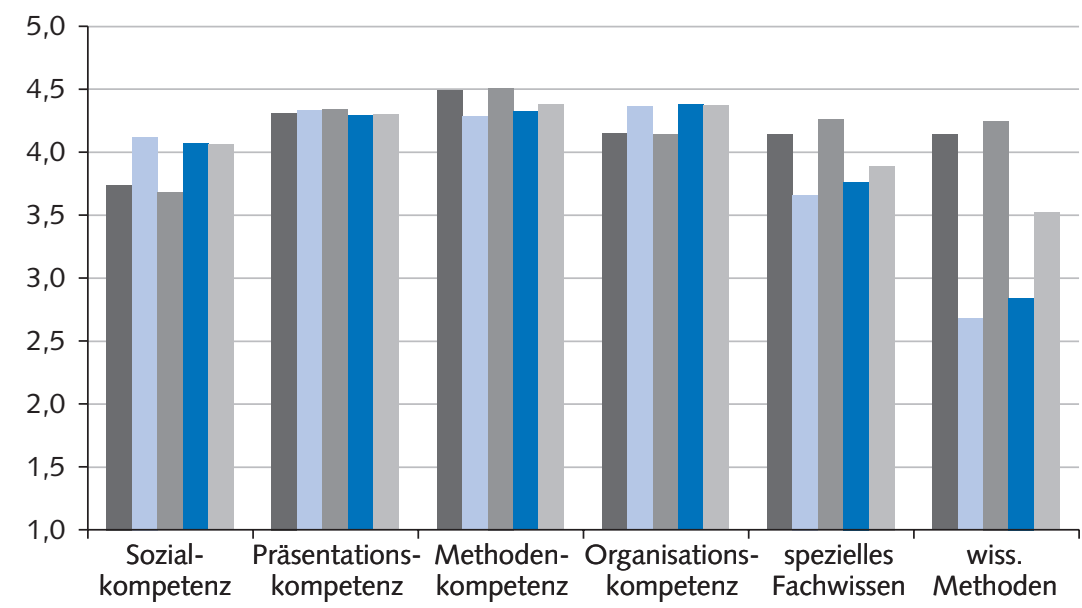

$$
\begin{aligned}
& \text { - Promovierende in angemessener Beschäftigung } 2001 \\
& \text { Angemessen Beschäftigte ohne Promotion } 2001 \\
& \text { Promovierende in angemessener Beschäftigung } 2005 \\
& \text { Angemessen Beschäftigte ohne Promotion } 2005 \\
& \text { Bachelorabsoventen } 2005
\end{aligned}
$$

1) gespiegelte Skala von 1 bis 5 (in geringem bis hohem Maße vorhanden).

Quelle: Darstellung des Autors.

WSI MITTEILUNGEN

gruppen recht nahe beieinander liegen. Hinsichtlich der geringeren Anforderungen vor allem an die Sozialkompetenzen Promovierender ist zu erwarten, dass diese nach Abschluss der Promotion ähnlich hohen Anforderungen ausgesetzt sein werden. Ergänzende Analysen mithilfe der zweiten Befragung des Jahrgangs von 2001 etwa fünf Jahre nach Studienabschluss weisen zumindest auf deskriptiver Ebene auf eine Nivellierung der Differenzen zwischen Promovierten und NichtPromovierten hin.

Schließlich ist noch einmal die Frage nach den Veränderungen im Verhältnis zwischen hochschulisch vermittelten und den für die berufliche Tätigkeit erforderlichen Kompetenzen aufzugreifen. Trotz der Abkehr eines Großteils der Absolventen vom Wissenschaftssystem scheint die wissenschaftsbezogene Qualifizierungsfunktion in den traditionellen universitären Studiengängen in Anbetracht der geringeren Defizite bei den bereichsspezifischen Fachkompetenzen mehr Gewicht (gehabt) zu haben als eine praxisbezogene, auf den Arbeitsmarkt gerichtete Qualifizierung. Auch die Ergebnisse früherer Analysen weisen darauf hin, dass Praxisbezüge innerhalb der Lehrveranstaltungen zwar positiven Einfluss auf Berufseinstiege haben; der Einbezug dieser Aspekte wird von den Absolventen jedoch eher als defizitär wahrgenommen. Jene Aspekte hingegen, die sich auf die Vermittlung wissenschaftlicher Arbeitsweisen beziehen, werden deutlich besser eingeschätzt und üben zugleich einen positiven Einfluss auf die Entscheidung zur wissenschaftlichen Weiterqualifizierung aus (Grotheer 2009; S. 257f.). Zusätzliche Schlüsselkompetenzen werden hingegen vorrangig über fachnahe Beschäftigung außerhalb des Fachstudiums erworben. In den reformierten Studiengängen scheint sich die Vermittlung von Schlüsselkompetenzen jedoch zumindest partiell verbessert zu haben.

\section{Fazit}

Mit der demografischen Entwicklung und der Reformierung von Studiengängen sind zweierlei Perspektiven gegeben, die Hypothesen im Hinblick auf die quantitative Entwicklung des Angebotes an hochqualifizierten Arbeitskräften und insbesondere der potenziellen Übergän- 
ge in Promotionen zur Sicherstellung der Innovationsfähigkeit zulassen. Bei konstanter Bildungsbeteiligung wird sich die demografische Entwicklung erst nach 2013 negativ auf die Zahl der Studienberechtigten und mit entsprechender zeitlicher Verzögerung auf die Zahl der Promovierenden auswirken. Offener ist hingegen die Frage, welche Wirkungen zunehmende Anteile von Bachelorabschlüssen, die nicht zur Promotion berechtigen, auf die quantitative Entwicklung der Übergänge in Promotionen haben werden. Problematisch sind zum einen hohe Abbrecherquoten in den reformierten Studiengän- gen. Zum anderen reduziert sich durch Bachelorabsolventen, die im Anschluss kein Masterstudium aufnehmen, die Zahl der potenziellen Promotionen. Zwar wird die Qualifizierung für die Forschung in vielen Masterstudiengängen als eines der Ziele definiert, sodass innerhalb dieser auch höhere Promotionsquoten erreicht werden sollten. Gleichzeitig muss jedoch die Lücke hinsichtlich der Forschungsorientierung und der Fachkompetenzen, die im Bachelorstudium aufgrund des höheren Stellenwerts von Praxisbezügen entsteht, im Masterstudium zunächst geschlossen werden. Für die Promovierenden konnte gezeigt werden, dass deren Kompetenzanforderungen einen Mix aus Schlüsselund Fachkompetenzen bilden, wobei den Schlüsselkompetenzen ein ähnlich hoher Stellenwert wie den Fachkompetenzen beizumessen ist. Positiv fällt dabei auf, dass einige der Schlüsselkompetenzen durch die Studiengestaltung der Bachelorstudiengänge stärker gefördert werden. Wenngleich die Anforderungen an die Sozialkompetenzen Promovierender zunächst geringer erscheinen, ist es sehr wahrscheinlich, dass Promovierte in der späteren post-doc-Phase höhere Anforderungen zu erwarten haben.

\section{LITERATUR}

Baethge, M./Baethge-Kinsky, V. (1998): Jenseits von Beruf und Beruflichkeit? Neue Formen von Arbeitsorganisation und Beschäftigung und ihre Bedeutung für eine zentrale Kategorie gesellschaftlicher Integration, in: Mitteilungen aus der Arbeitsmarkt- und Berufsforschung (MittAB)

31, S. 438-460

Berning, E./Falk, S. (2005): Das Promotionswesen im Umbruch, in: Beiträge zur Hochschulforschung 27, S. 49-72

Blossfeld, H. P. (1989): Kohortendifferenzierung und Karriereprozeß. Eine Längsschnittstudie über die Veränderung der Bildungs- und Berufschancen im Lebenslauf, Frankfurt/New York

Briedis, K. (2007): Übergänge und Erfahrungen nach dem Hochschulabschluss. Ergebnisse der HIS-Absolventenbefragung des Jahrgangs 2005. HIS: Forum Hochschule 13, Hannover

Dietrich, H./Abraham, M. (2005): Eintritte in den Arbeitmarkt, in: Abraham, M./Hinz, T. (Hrsg.): Arbeitsmarktsoziologie. Probleme, Theorien, empirische Befunde, Wiesbaden, S. 69-98

Fabian, G./Briedis, K. (2009): Aufgestiegen und erfolgreich. Ergebnisse der dritten Absolventenbefragung des Jahrgangs 1997 zehn Jahre nach dem Examen, HIS: Forum Hochschule 2, Hannover

Falk, S./Sackmann, R./Struck, O./Weymann, A./Windzio, M./ Wingens, M. (2000): Gemeinsame Startbedingungen in Ost- und West? Risiken beim Berufseinstieg und deren Folgen im weiteren Erwerbsverlauf, Arbeitspapier 65, SFB186, Bremen

Gensch, S. K./Schindler, G. (2003): Bachelor- und Masterstudiengänge an den staatlichen Hochschulen in Bayern, Bayrisches Staatsinstitut für Hochschulforschung und Hochschulplanung, München
Goudswaard, A./de Nanteuil, M. (2000): Flexibility and Working Conditions: A Qualitative and Comparative Study in Seven EU Member States. A Summary, European Foundation for the Improvement of Living and Working Conditions, Dublin

Grotheer, M. (2008): Beschäftigungsstabilität und -sicherheit in Westdeutschland. Entwicklungsdynamik und Folgen für die soziale Ungleichheit, in: Köhler, C./Struck, O./Grotheer, M./Krause, A./Krause, I./ Schröder, T.: Offene und geschlossene Beschäftigungssysteme. Determinanten, Risiken und Nebenwirkungen, Wiesbaden, S. 65-113

Grotheer, M. (2009): Studienqualität, berufliche Einstiege und Berufserfolg von Hochschulabsolventinnen und Hochschulabsolventen. Eine Analyse der Arbeitsmarktchancen der Absolventenkohorten von 1997, 2001 und 2005, in: Hochschul-Informations-System (Hrsg.): Perspektive Studienqualität. Themen und Forschungsergebnisse der HIS-Fachtagung "Studienqualität“, WBV, Bielefeld, S. 244-262

Grotheer, M. (2010): Berufliche Einstiege und Berufserfolg von Hochschulabsolventinnen und Hochschulabsolventen. Forschungsbericht zur Entwicklung der Arbeitsmarktchancen der Absolventenkohorten von 1997, 2001 und 2005, HIS: Forum Hochschule, Hannover (im Erscheinen)

Heublein, U./Hutzsch, C./Schreiber, J./Sommer, D./Besuch, G. (2009): Ursachen des Studienabbruchs in Bachelor- und herkömmlichen Studiengängen. Ergebnisse einer bundesweiten Befragung von Exmatrikulierten des Studienjahres 2007/08, HIS Projektbericht, Hannover Isserstedt, W./Middendorff, E./Fabian, G./Wolter, A. (2007): Die wirtschaftliche und soziale Lage der Studierenden in der Bundesrepublik 
Deutschland 2006. 18. Sozialerhebung des Deutschen Studentenwerks, durchgeführt durch HIS Hochschul-Informations-System, Hannover, BWH GmbH, Medien Kommunikation

Jäger, P. (2001): Der Erwerb von Kompetenzen als Konkretisierung der Schlüsselqualifikationen - Eine Herausforderung an Schule und Unterricht. Dissertation, Universität Passau. online: http://www.opus-bayern. de/uni-passau/volltexte/2003/17/pdf/jaeger.pdf (Zugriff 1.3.2010) Kerst, C./Schramm, M. (2008): Der Absolventenjahrgang 2000/2001 fünf Jahre nach dem Hochschulabschluss. Berufsverlauf und aktuelle Situation, HIS: Forum Hochschule 10, Hannover

Krull, W. (2008): Die Excellenzinitiative und ihre Folgen. Deutsche Hochschulen vor neuen Herausforderungen, in: Kehm, B. M. (Hrsg.): Hochschule im Wandel. Die Universität als Forschungsgegenstand, Frankfurt/New York, S. 243-251

Leemann, R. J. (2002): Chancenungleichheiten im Wissenschaftssystem. Wie Geschlecht und soziale Herkunft Karrieren beeinflussen, Zürich

OECD (1998): Getting started, settling in: the transition from education to labour market, in: OECD: Employment Outlook, Paris, S. 81-122
Orth, H. (1999): Schlüsselqualifikationen an deutschen Hochschulen. Konzepte, Standpunkte und Perspektiven, Neuwied u. a.

Picot, A./Reichwald, R./Wigand, R. T. (2001): Die grenzenlose Unternehmung. Information, Organisation und Management, Wiesbaden Schaeper, H./Briedis, K. (2004): Kompetenzen von Hochschulabsolventinnen und Hochschulabsolventen, berufliche Anforderungen und Folgen für die Hochschulreform, HIS-Kurzinformation A6/2004, Hannover Schaeper, H./Wolter, A. (2008): Hochschule und Arbeitsmarkt im Bologna-Prozess. Der Stellenwert von „Employability“ und Schlüsselkompetenzen, in: Zeitschrift für Erziehungswissenschaft (ZfE) 11, S. 607-625

Weinert, F. E. (1998): Vermittlung von Schüsselqualifikationen, in: Matalik, S./Schade, D. (Hrsg.): Entwicklungen in Aus- und Weiterbildung: Anforderungen, Ziele, Konzepte, Baden-Baden, S. 23-43

Wissenschaftsrat (1999): Stellungnahme zum Verhältnis von Hochschulausbildung und Beschäftigungssystem, Drs. 4099/99, Würzburg Wissenschaftsrat (2005): Entwicklung der Fachstudiendauer an Universitäten von 1999 bis 2003, Drs. 6825, Köln

Wissenschaftsrat (2006): Empfehlungen zur Entwicklung und Förderung der Geisteswissenschaften in Deutschland, Drs. 7068, Berlin 